\title{
Control of polarization rotation in nonlinear propagation of fully-structured light
}

\author{
Christopher J. Gibson, Patrick Bevington, Gian-Luca Oppo and Alison M. Yao ${ }^{1}$ \\ ${ }^{I}$ SUPA and Department of Physics, University of Strathclyde, Glasgow G4 ONG, Scotland, U.K.
}

\begin{abstract}
Knowing, and controlling, the spatial polarization distribution of a beam is of importance in applications such as optical tweezing, imaging, material processing and communications. Here we show how the polarization distribution is affected by both linear and nonlinear (self-focussing) propagation. We derive an analytical expression for the polarization rotation of fully-structured light (FSL) beams during linear propagation and show that the observed rotation is due entirely to the difference in Gouy phase between the two eigenmodes comprising the FSL beams, in excellent agreement with numerical simulations. We also explore the effect of cross-phase modulation due to self-focusing (Kerr) nonlinearity and show that polarization rotation can be controlled by changing the eigenmodes of the superposition, and physical parameters such as the beam size, the amount of Kerr nonlinearity and the input power. Finally, we show that by biasing cylindrical vector (CV) beams to have elliptical polarization, we can vary the polarization state from radial through spiral to azimuthal using nonlinear propagation.
\end{abstract}

\section{INTRODUCTION}

Vector, or fully structured light (FSL), beams [1--3] have attracted increasing attention for a number of applications. These beams consist of a vector superposition of two scalar orbital angular momentum (OAM) carrying Laguerre-Gaussian (LG) eigenmodes with orthogonal polarizations. The resultant beam has non-uniform spatial intensity, phase and polarization distributions. The ability to control both the spatial intensity and the polarization distribution of the optical field is of use in material processing [4]), in STED and confocal microscopy [5-8], in optical trapping and manipulation [9. 10], in atomic state preparation, manipulation, and detection [9, 11, 12], in optical communications [13, 14] and even classical entanglement [15-17]. Additionally, novel focussing properties associated with particular polarization distributions can lead to tighter focussing [18] and strong axial field components that are of use in microscopy [5, 6], optical trapping [9], and as a mechanism for linear accelerators [19]. It is therefore important to understand how the spatial intensity and polarization distributions can be affected - and potentially controlled - by linear and nonlinear beam propagation.

It has been shown previously that the polarization distribution of a lower-order Poincaré beam of $\ell_{L}=0, \ell_{R}=1$ and net $\mathrm{OAM}=1$, undergoes a rigid rotation of $\pi / 2$ as it propagates linearly from the waist plane to the far field zone [2]. Here we derive an analytical expression for the polarization rotation of any FSL beam during linear propagation. We show that the observed rotation is due entirely to the difference in the Gouy phase between the two eigenmodes comprising the FSL beams and explain apparent inconsistencies in the rotation of different polarization distributions. Our results are in excellent agreement with numerical simulations.

For many applications it is useful to retain a desired intensity and/or polarization distribution, for example for modern optical technologies and high-power laser systems [20]. Beams carrying OAM are of particular interest due to their potential to carry an increased information content [13]. Although the effect of beam spreading due to diffraction during linear propagation can be mitigated, and in some cases exactly balanced, by using a self-focusing (Kerr) nonlinear medium, OAM beams are known to fragment into multiple (twice the OAM) soliton peaks during nonlinear propagation [21, 22]. It has been shown that this fragmentation can be inhibited, without altering the nonlinear confinement, by using vector beams instead of scalar beams [14, 23-25]. Indeed, it has been shown that cylindrical vector $(\mathrm{CV})$ beams can propagate in a saturating Kerr nonlinear medium with no change to their spatial profile or their axially symmetric polarization distribution for much longer distances than the equivalent scalar beams [14]. Similar spatial confinement can be seen for FSL beams, though in this case the nonlinear propagation will affect the rotation of the polarization structure.

Here we investigate how nonlinear propagation affects the polarization distribution. We show how the magnitude and direction of the polarization rotation is affected by the spatial overlap between the two eigenmodes, and how it can be controlled by modifying the effective nonlinearity via physical parameters such as the input power, the temperature of the sample, and the size of the FSL beam waist.

In addition to self-focusing, a Kerr medium also induces a cross-phase modulation between the two eigenmodes of the vector beam. We show that as the coupling tries to maximise the spatial overlap, the rotation of the polarization structure is no longer rigid. Note that although we do not propagate our beams to the point of fragmentation, one effect of this homogenisation is that the beams will fragment into equalsized solitons.

Finally, we show that the nonlinearity not only changes the amount of polarization rotation for beams with a net OAM, but that it can also induce a rotation in beams with zero net OAM if there is an amplitude difference between the two modes. By biasing cylindrical vector (CV) beams such that they have $\mathrm{el}$ liptical polarization, we can vary the polarization distribution from radial through spiral to azimuthal during nonlinear propagation. 


\section{FULLY-STRUCTURED LIGHT BEAMS}

Fully-Structured Light (FSL) beams, or Poincaré beams, are are constructed from a vector superposition of two scalar orbital angular momentum (OAM) carrying spatial transverse eigenmodes with orthogonal polarizations:

$$
\vec{E}(r, \phi, z)=E_{L}(\rho, \phi, z) \overrightarrow{e_{l}}+E_{R}(\rho, \phi, z) \overrightarrow{e_{r}},
$$

with

$$
E_{L}=\cos (\gamma) L G_{L} ; E_{R}=e^{i \beta} \sin (\gamma) L G_{R},
$$

(1) where $I$ is the intensity and the subscripts $R, L$ denote the

where $\gamma$ and $\beta$ give the relative amplitudes and phase, respectively, of the two modes. Throughout this paper we have adopted the circular polarization basis and we assume that each of the spatial modes takes the form of a LaguerreGaussian beam with radial index $p=0$ [26]:

$$
\begin{aligned}
& L G_{0}^{\ell}(r, \phi, z)=\sqrt{\frac{2}{\pi|\ell| !}} \frac{1}{w(z)} \exp \left(\frac{-r^{2}}{w(z)^{2}}\right)\left(\frac{r \sqrt{2}}{w(z)}\right)^{|\ell|} \\
& \times \exp (i \ell \phi) \exp \left(\frac{i k r^{2} z}{2\left(z^{2}+z_{R}^{2}\right)}\right) \exp [-i(|\ell|+1) \eta(z)],
\end{aligned}
$$

where $\ell$ is the orbital angular momentum (OAM) index of the mode, $\phi$ is the azimuthal angle, $z_{R}=k_{0} w_{0}^{2} / 2$ is the Rayleigh range for a beam with waist $w_{0}$ and free-space wavenumber $k_{0}, w(z)=w_{0} \sqrt{1+z^{2} / z_{R}^{2}}$ describes the linear diffraction of the beam and $(|\ell|+1) \eta(z)$, with $\eta(z)=\tan ^{-1}\left(z / z_{R}\right)$, is the Gouy phase [27]. If either $E_{L}$ or $E_{R}$ is zero, then the resultant beam is a scalar LG mode with spatially uniform rightor left-handed circular polarisation, respectively. If the two modes have equal amplitude and the same OAM, the resultant beam will have spatially uniform linear polarisation. If they have equal amplitude and equal but opposite OAM, however, the resultant cylindrical vector (CV) beam [1, 3] can have spatially varying linear polarization distributions that are radial, azimuthal or spiral, depending on the phase difference $\beta$. The polarization of the beam will be axially symmetric about the beam's propagation axis and span the equator of the Poincare sphere. If the two modes have different magnitudes of OAM, such that the resultant FSL beam carries a net OAM, the polarization will vary in both the angular and radial coordinates and covers all polarization states on the Poincaré sphere [2]. Note that we are working in the paraxial regime throughout, which is an excellent approximation as we are considering beam sizes that are larger than the wavelength, and that our analysis is based on vector superpositions of Laguerre-Gaussian modes, which are solutions of the paraxial wave equation in cylindrical coordinates [26]. Extension to the non-paraxial regime is non-trivial as in this case the beam polarization is a local property and changes on propagation [28]. Moreover, during propagation, a non-paraxial vortex mode will acquire a non-integrable topological phase with no simple analytical form [29]. This tends to the Gouy phase, on which our analytical calculations are based, when the paraxial approximation is valid. right and left circular components, $E_{R} \equiv\left(E_{x}-i E_{y}\right) / \sqrt{2}$, and

In order to map the polarization distribution on the transverse plane we use the Stokes parameters that, in the circular polarization basis, are given by [30]

$$
\begin{array}{rll}
S_{0}=I=\left|E_{R}\right|^{2}+\left|E_{L}\right|^{2} & ; & S_{1}=2 \operatorname{Re}\left(E_{R}^{*} E_{L}\right), \\
S_{2}=2 \operatorname{Im}\left(E_{R}^{*} E_{L}\right) \quad ; & S_{3}=\left|E_{R}\right|^{2}-\left|E_{L}\right|^{2},
\end{array}
$$
$\left.E_{L} \equiv\left(E_{x}+i E_{y}\right) / \sqrt{2}\right)$. We can then calculate the ellipticity,
$\chi$, and orientation, $\psi$, of the polarization at each point on the transverse plane using [31]

$$
\frac{S_{1}}{S_{0}}=\cos 2 \chi \cos 2 \psi ; \frac{S_{2}}{S_{0}}=\cos 2 \chi \sin 2 \psi ; \frac{S_{3}}{S_{0}}=\sin 2 \chi,
$$

which give:

$$
\begin{aligned}
& \chi=\frac{1}{2} \sin ^{-1}\left(\frac{S_{3}}{S_{0}}\right)=\frac{1}{2} \sin ^{-1}\left(\frac{\left|E_{R}\right|^{2}-\left|E_{L}\right|^{2}}{\left|E_{R}\right|^{2}+\left|E_{L}\right|^{2}}\right), \\
& \psi=\frac{1}{2} \tan ^{-1}\left(\frac{S_{2}}{S_{1}}\right)=\frac{1}{2} \tan ^{-1}\left(\frac{\operatorname{Im}\left(E_{R}^{*} E_{L}\right)}{\operatorname{Re}\left(E_{R}^{*} E_{L}\right)}\right) .
\end{aligned}
$$

Typical examples of polarization distributions of FSL beams are given in Fig. 1 .
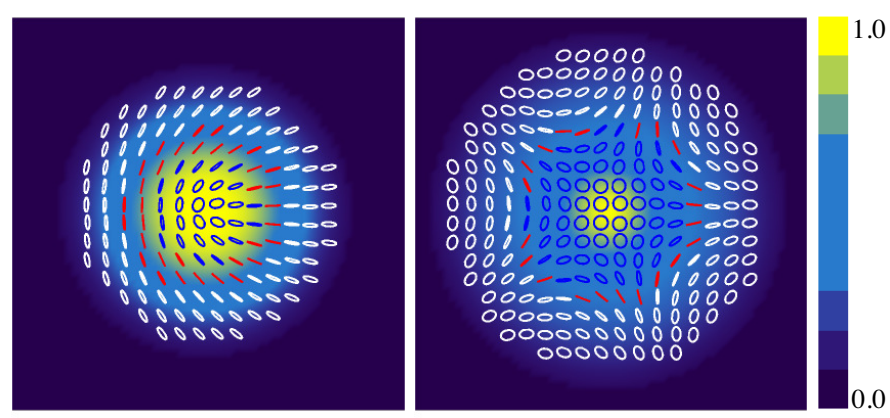

FIG. 1: Polarization distributions at the beam waist for FSL beams with (left) "lemon" polarization, $\ell_{R}=1, \ell_{L}=0$, and (right) "web" polarization, $\ell_{R}=-3, \ell_{L}=0$, polarization at the beam waist (propagation distance $z=0$ ). White, red, blue lines correspond to right circular, linear, left circular polarization, respectively.

\section{Polarization rotation during linear propagation}

We can calculate the orientation of the polarization at any transverse point in the field by using Eqs. 2 and 3 to re-write, see Eq. 6. in terms of the constituent modes:

$$
\psi(z)=\frac{1}{2}\left[\left(\ell_{L}-\ell_{R}\right) \phi-\left(\left|\ell_{L}\right|-\left|\ell_{R}\right|\right) \eta(z)-\beta\right] .
$$

From this it is clear that the initial orientation of the polarization at any point, $\psi(0)=\frac{1}{2}\left[\left(\ell_{L}-\ell_{R}\right) \phi-\beta\right]$, depends only on the difference in OAM of the two eigenmodes, together with any fixed initial phase difference, $\beta$ and is independent of 
$z$. We note that in the case of scalar beams, where either $E_{L}$ or $E_{R}$ is zero, the polarization is right- or left-circular and so there is no direction of polarisation orientation.

As the beam propagates from $z=0$, the net polarization rotation is simply

$$
\Delta \psi(z)=\psi(z)-\psi(0)=\frac{1}{2} \Delta\left|\ell_{R, L}\right| \eta(z),
$$

where $\Delta\left|\ell_{R, L}\right|=\left|\ell_{R}\right|-\left|\ell_{L}\right|$. This depends only on the difference in the magnitudes of the OAM of the two modes and the Gouy phase $\eta(z)$. Note that both the polarization orientation and rotation are independent of the amplitudes of the two modes and that the amount of rotation is the same for every point in the transverse plane. This reduces to

$$
\Delta \psi=\frac{\pi}{8} \Delta\left|\ell_{R, L}\right|
$$

for a beam propagating from a waist plane $z=0$ through a Rayleigh range $z=z_{R}$ and asymptotes to

$$
\Delta \psi=\frac{\pi}{4} \Delta\left|\ell_{R, L}\right|
$$

as the beam propagates into the far field (since $\tan ^{-1}\left(z / z_{R}\right) \rightarrow$ $\pi / 2$ as $z \rightarrow \infty)$.

Fig. 2 shows the polarization rotation during linear propagation given by 8 for a number of FSL beams with different values of $\Delta\left|\ell_{R, L}\right|$ propagating from a beam waist $z=0$ to $z=20 z_{R}$, solid lines. As expected the rotation asymptotes to $\frac{\pi}{4} \Delta\left|\ell_{R, L}\right|$. We note that this result depends only on the difference in the magnitudes of the two OAM eigenmodes, not on their particular values, and that there is no polarization rotation for cylindrical vector beams as both modes have the same magnitude of OAM and hence experience the same Gouy phase. Fig. 3 shows the polarization distributions at

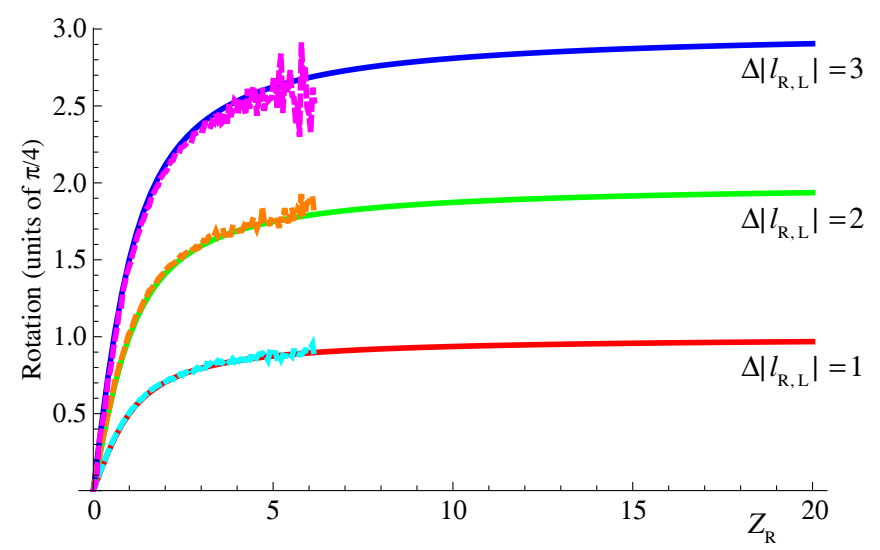

FIG. 2: Polarization rotation calculated using 8 for linear propagation from a beam waist $z=0$ to the far-field $z=20 z_{R}$ for FSL beams, solid lines. Dashed lines give the equivalent numerical results for $z=0$ to $z=6 z_{R}$ using 12 with $\mu=0 . \Delta\left|\ell_{R, L}\right|=1$ (red, cyan), 2 (green, orange), 3 (blue, magenta). Note that rotation is plotted in units of $\pi / 4$ to clarify the asymptotic behaviour.

$z=20 z_{R}$ for beams with (left) $\ell_{R}=1, \ell_{L}=0 \Rightarrow \Delta\left|\ell_{R, L}\right|=1$ and (right) $\ell_{R}=-3, \ell_{L}=0 \Rightarrow \Delta\left|\ell_{R, L}\right|=3$. Note that diffraction corresponds to a global scaling according to $w(z)$ that has been neglected for clarity. Comparing these to the
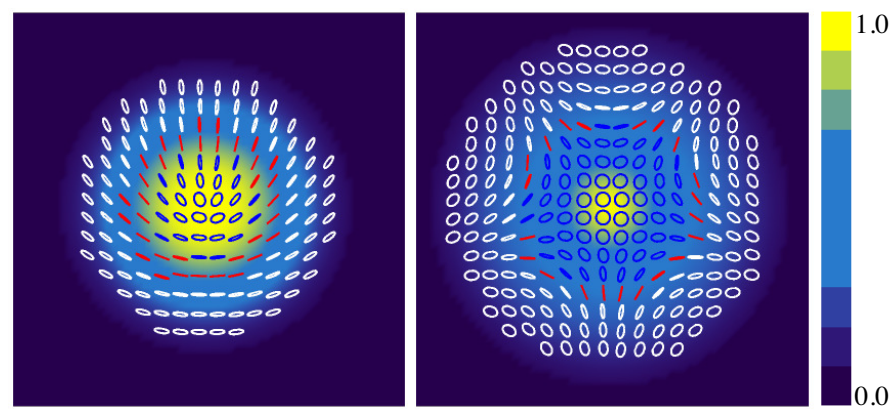

FIG. 3: Polarization distributions at $z=20 z_{R}$ for FSL beams with (left) $\ell_{R}=1, \ell_{L}=0$, and (right) $\ell_{R}=-3, \ell_{L}=0$. White, red, blue lines correspond to right circular, linear, left circular polarization, respectively. Note that beam expansion due to propagation has no effect on the polarization distribution and so has been neglected for clarity.

initial polarization distributions at the beam waist, Fig. 1, it appears that the polarization distribution on the left has rotated rigidly anti-clockwise by $\pi / 2$, as observed in [2], while the polarization distribution on the right has rotated rigidly anti-clockwise by $\pi / 4$. Although these results seem to contradict the predicted rotations of $\pi / 4$ and $3 \pi / 4$, respectively, on closer inspection one can see that while the polarization at each point on the transverse plane has indeed as predicted by 8 , the apparent rotation of the polarization structure as a whole depends on the rotational symmetry of the pattern.

We also model linear propagation of the FSL beams numerically using the paraxial equation normalized to dimensionless quantities, $\rho=r / w_{0}$ and $\zeta=z /\left(2 z_{R}\right)$, where $w_{0}$ is the beam waist and $z_{R}$ is the Rayleigh range of the beam [21-23]

$$
\frac{\partial E_{L, R}}{\partial \zeta}=\frac{i}{2} \nabla_{\perp}^{2} E_{L, R}
$$

Here $E_{L, R}$ are the left- and right-circularly polarized beams and $\nabla_{\perp}^{2}$ is the Laplacian in the transverse $(x, y)$ plane. As Fig. 2 shows, we find excellent agreement between the analytical and numerical results until the beam diffraction is too large to accurately track the polarization rotation (approximately $z=$ $\left.5 z_{R}\right)$. Note that the effect of diffraction is more pronounced for higher-order FSL beams, resulting in increased noise as the propagation distance increases. Propagation of radial and the azimuthal components of a paraxial beam along the optical axis of a uniaxial anisotropic crystal has been investigated in [32] and references within.

\section{NONLINEAR PROPAGATION}

As mentioned earlier, it is possible to counteract the effect of diffraction and to control the spatial profile of the beam using a self-focussing (Kerr) nonlinearity. The usual fragmenta- 
tion of scalar vortex (OAM) beams in Kerr media can be inhibited by using vector vortex or FSL beams. It has been shown that cylindrical vector (CV) beams can additionally propagate with no change to their axially symmetric polarization distribution, while lower order $(\ell=0,1)$ FSL beams experience a polarization rotation [14]. We note that azimuthally polarized, spatial, dark soliton solutions of Maxwell's equations without OAM have been demonstrated in [33].

In order to investigate the effect of nonlinear propagation on the polarization we numerically simulated propagation of FSL beams through a Kerr medium using two coupled $(2+1)$ dimensional nonlinear Schrödinger equations with saturable self-focusing nonlinearity under the slowly varying envelope and paraxial approximations and normalized to dimensionless quantities, $\rho=r / w_{0}$ and $\zeta=z /\left(2 z_{R}\right)$

$$
\frac{\partial E_{L, R}}{\partial \zeta}=\frac{i}{2} \nabla_{\perp}^{2} E_{L, R}+i \mu \frac{\left|E_{L, R}\right|^{2}+2\left|E_{R, L}\right|^{2}}{1+\sigma\left(\left|E_{L, R}\right|^{2}+2\left|E_{R, L}\right|^{2}\right)} E_{L, R}
$$

where the parameters of importance are the nonlinear parameter, $\mu$, and the saturation parameter, $\sigma$, given by:

$$
\mu=\frac{2 k_{0}^{2} n_{2} P_{0}}{3 n_{0}} ; \quad \sigma=\frac{4 P_{0}}{3 I_{\text {sat }} w_{0}^{2}},
$$

where $k_{0}$ is the free-space wavenumber, $n_{0}$ and $n_{2}$ are the linear and nonlinear refractive indices $\left(n_{2}>0\right.$ for self-focusing), $I_{\text {sat }}$ is the saturation intensity, and $P_{0}$ is the power of the incident laser beam.

If one neglects the beam rescaling due to diffraction, we can see from Eq. 12 that the first effect of the medium is an additional phase shift $\phi_{R, L}^{N L}$ to the orthogonal modes proportional to the nonlinear term. This results in an additional rotation $\Delta \psi_{N L}(z) \propto\left(\phi_{L}^{N L}-\phi_{R}^{N L}\right)$. As the nonlinear phase shift depends on the spatial intensity of the two modes and on the cross phase modulation, we expect the nonlinear rotation to be dependent on the magnitude of the OAM of each mode (and not just the difference in the net values). We also expect the rotation to be spatially-dependent, except for the CV beams which have the same magnitude of OAM and hence the same spatial profile. We show the effect of the spatial dependence in more detail in Fig. 6 but the fact that the polarization rotation is spatially dependent then begs the question of how to measure it. An overall sense of the polarization rotation can be found either by considering a point that remains at the peak intensity of the FSL beam, as shown in Fig. 6(green line), or by taking an average over a number of points across the beam. In cases where both eigenmodes have non-zero OAM these two approaches are in good agreement. However, when one of the eigenmodes is a gaussian, the transverse position of the peak changes a lot during propagation and so the averaging method gives the best sense of the rotation behaviour. For that reason we use this method for all of our nonlinear results, unless otherwise stated. Obviously for more exact measurements, the rotation can be measured and compared at many points across the beam. In the simulations reported below, we have selected $P_{0}=7.4 \mathrm{~mW}, I_{\text {sat }}=5 \mathrm{~W} \mathrm{~cm}{ }^{-2}, n_{2}=8 \times 10^{-6} \mathrm{~cm}^{2} / \mathrm{W}$ and $\lambda=780 \mathrm{~nm}$ that reproduce the experimental configuration given in [14]. We use a beam waist of $100 \mu \mathrm{m}$ throughout (unless explicitly stated otherwise), corresponding to a Rayleigh range of approx. $4 \mathrm{~cm}$. We performed numerical integrations of the propagation Eqs. 12, using the split-step method with fast Fourier transforms.

\section{Comparison with the linear case}

To investigate the effect of the propagation in the nonlinear medium we first numerically integrated Eq. 12 for FSL beams with $\ell_{R}= \pm 1, \pm 2, \pm 3$ and $\ell_{L}=0$ giving $\Delta\left|\ell_{R, L}\right|=1,2,3$, respectively, with the parameters given above. Note that in order to avoid the numerical errors that occur when the beams have diffracted significantly, we present only results for propagation up to $3 z_{R}$ throughout the remainder of the paper.

As Fig 4 shows, in the nonlinear medium the polarization rotation no longer asymptotes but instead increases almost linearly with propagation distance. For completeness, we note that there is again no rotation for cylindrical vector beams as both modes have the same magnitude of OAM and the same spatial intensity and so that they experience the same Gouy and nonlinear phase shifts.

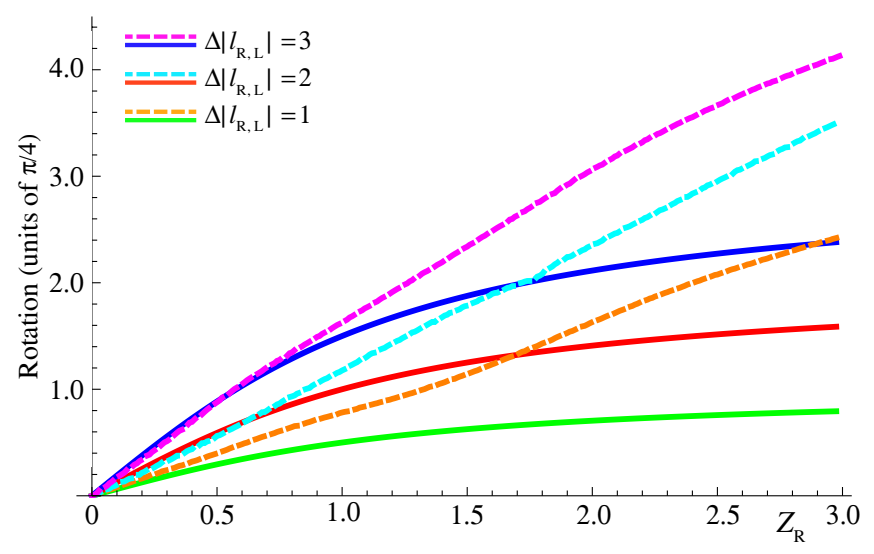

FIG. 4: Polarization rotation during nonlinear propagation from a beam waist $z=0$ to $z=3 z_{R}$ using 12 with $\mu=257.5$ and $\sigma=19.8$ for FSL beams with $\ell_{R}= \pm 1, \pm 2, \pm 3$ and $\ell_{L}=0$ (dashed lines). Solid lines give the corresponding analytical results for linear propagation using $8 \Delta\left|\ell_{R, L}\right|=1$ (red, cyan), 2 (green, orange), 3 (blue, magenta).

\section{Effect of beam composition}

Unlike in the linear case, where the polarization rotation depends solely on the difference in the magnitudes of the OAM of the two eigenmodes, in the nonlinear medium the phase shift, and hence polarization rotation, depends on the spatial overlap of the eigenmodes and the cross-phase modulation between them. To demonstrate this we repeated our simulations for a number of different mode superpositions with 
the same difference in the Gouy phase, i.e. a fixed value of $\Delta\left|\ell_{R, L}\right|=\left|\ell_{R}\right|-\left|\ell_{L}\right|$.

In Fig. 5 we show the polarization rotation for $\Delta\left|\ell_{R, L}\right|=2$. The red line is the analytical linear result for all superpositions, as in Fig. 2, while the cyan line is the nonlinear result for a beam with $\ell_{R}=2 ; \ell_{L}=0$, as in Fig. 4. The dashed green line shows the equivalent result but for a beam with $\ell_{R}=3 ; \ell_{L}=-1$ (green). Note that similar behaviour is seen for the beam with $\ell_{R}=4 ; \ell_{L}=-2$ but this is omitted for clarity. We see a significant change in the behaviour of the polarization rotation between the lowest-order FSL beam $\ell_{L}=0$ and the higher-order beams $\left|\ell_{L}\right|>0$. In particular we note that the higher-order beam experiences less polarization rotation overall: there are regions during the propagation where the polarization rotation seems to stop, or even change direction, and the rotation appears to asymptote with increasing propagation distance. There are two important phenomena af-

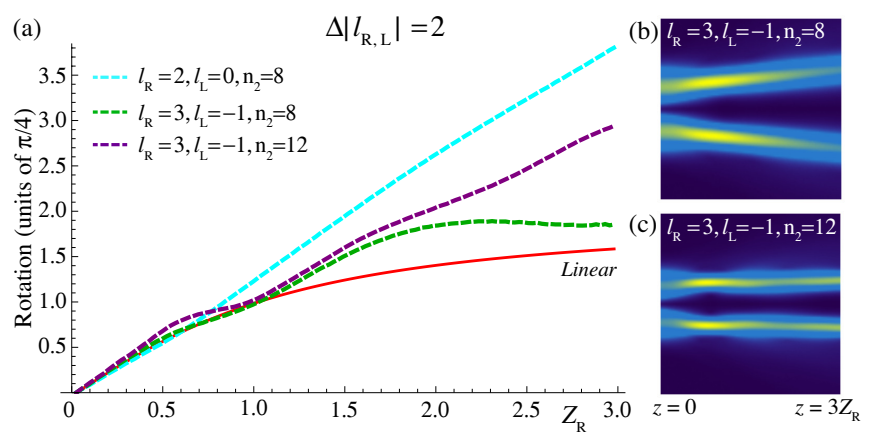

FIG. 5: (a) Polarization rotation during nonlinear propagation from a beam waist $z=0$ for 3 Rayleigh ranges, $z=3 z_{R}$, for FSL beams with beam waist $w_{0}=100 \mu \mathrm{m}$ and $\Delta\left|\ell_{R, L}\right|=2$. Cyan line corresponds to $\ell_{R}= \pm 2, \ell_{L}=0$ at low nonlinearity $\left(n_{2}=8\right)$. Dashed lines correspond to $\ell_{R}= \pm 3, \ell_{L}= \pm 1$ at $n_{2}=8$ (green) and $n_{2}=12$ (purple). Red line is the equivalent linear result for all superpositions. (b) \& (c) Cross-section of the spatial intensity during propagation for the FSL beams of $\ell_{R}= \pm 3, \ell_{L}= \pm 1$ at $n_{2}=8$ (top) and $n_{2}=12$ (bottom)

fecting the behaviour of the polarization rotation: the intensity of the modes and their spatial overlap. In Fig. 5 we plot the cross-section of the beam intensity of the $\ell_{R}=3, \ell_{L}=-1$ mode at two different values of the nonlinearity, $n_{2}=8$ (top) and $n_{2}=12$ (bottom). It is clear that there is still significant diffraction for $n_{2}=8$, and the corresponding reduction in intensity results in less nonlinear phase shift and leads to the asymptotic behaviour of the rotation (green line in Fig. 5 (a)). At $n_{2}=12$, however, the beam is quite well-confined and there is also a more consistent mode overlap. In this case we almost recover the rotation experienced by the lowest order mode (dashed purple line in Fig. 5a).

As mentioned earlier, the nonlinear polarization rotation also depends on the cross-phase modulation and hence on the spatial overlap of the eigenmodes. An overall sense of the behaviour of the polarization rotation can usually be found by tracking the polarization at point that remains at the peak intensity of the FSL beam. However, when one of the eigenmodes is a gaussian, the transverse position of the peak changes significantly during propagation and so a better method is to average the results over a number of points across the beam. For applications requiring more exact knowledge of the polarization state at each point on the transverse plane the polarisation rotation can be compared at various points across the beam. To demonstrate the effect of the cross-phase modulation term we consider a beam with eigenmodes of $\ell_{R}=3$ and $\ell_{L}=-1$. As the cross-phase modulation term is asymmetric unless $\ell_{R}=\ell_{L}$ and contains a saturating term, the mode sizes will oscillate during propagation with corresponding variations in the spatial overlap. The polarization rotation at each point will therefore depend on its location on the transverse plane. This is demonstrated in Fig. 6 for propagation of a FSL beam with $\ell_{R}=3 ; \ell_{L}=-1$ at low nonlinearity $\left(n_{2}=8\right)$. At the start of the propagation (see Fig. 6a), points on the outer edge of the beam (magenta) see more right-circularly polarized (RCP) light while points closer to the centre (blue) see more left-circularly polarized (LCP) light and thus their polarizations rotate in opposite directions. When the modes overlap exactly (Fig. 60) the modes experience the same nonlinear phase shift and so there is no net nonlinear rotation. If the beams remain "locked" like this there is a corresponding plateau in the rotation curve (see, for example, Fig. 5, green line). As the modes oscillate net left- or right-circularly polarized light can swap resulting in polarization rotation changing direction (Fig. 66). Note that as the beam continues to propagate the effect of the nonlinearity is to try to maintain the spatial overlap of the two modes. The "locked" beams then propagate with a constant small rotation depending on the overlap until they fragment into solitons [21, 22] (Fig. 61).
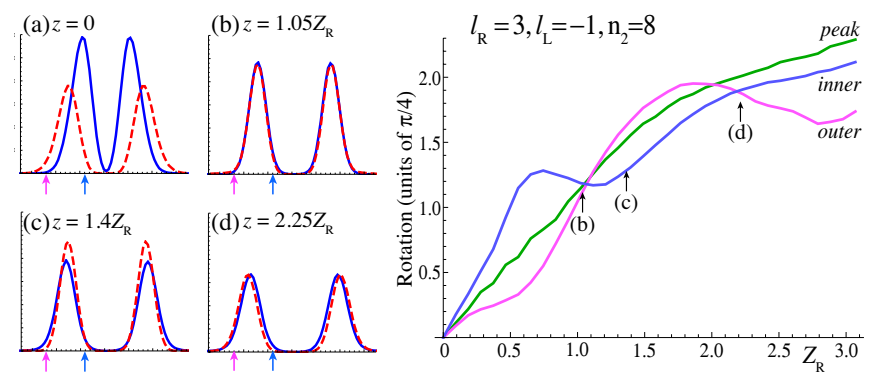

FIG. 6: Left: Cross-section of modes $\left(\ell_{R}=3\right.$, red, and $\ell_{L}=-1$, blue $)$ with beam waist $w_{0}=100 \mu \mathrm{m}$ showing the spatial overlap at various points during nonlinear propagation at low nonlinearity $\left(n_{2}=8\right)$. Right: Corresponding polarization rotation. Magenta and blue lines corresponds to points close to the outer edge and center of the FSL beam, respectively. Green line corresponds to the position of the peak intensity.

\section{Control of polarization rotation}

In addition to understanding how the state of polarization is affected by propagation, it may be useful to be able to control it. Linearly the polarization rotation can be controlled 
by choosing the eigenmodes of the FSL beam to give a specific difference in the magnitudes of the OAM $\left(\Delta\left|\ell_{R, L}\right|\right)$, as shown in Fig. 2, and by choosing the beam size to control the Rayleigh range.

Nonlinearly, the amount and direction of the nonlinear polarization rotation depends on the spatial overlap and intensity of the eigenmodes. These can be controlled both by the choice of eigenmodes (see Fig. 5) and by changing experimental parameters such as the power of the FSL beam, the temperature of the medium [14] and the size of the FSL beam to control the nonlinear parameter, $\mu$, and the saturation parameter, $\sigma$ (Eq. 13 and hence the confinement of the beam.

For example, Fig. 7 shows how the polarization rotation of the $\ell_{R}=1$ and $\ell_{L}=0$ beam changes as we increase the input power $P$. This increases both the nonlinear response and the saturation parameter leading to an enhanced polarization rotation at the output.

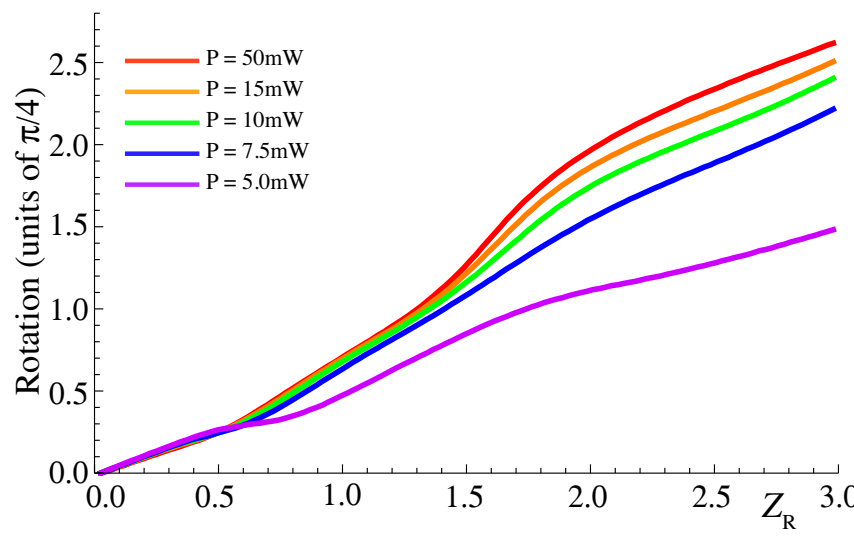

FIG. 7: Polarization rotation of $\ell_{R}=1$ and $\ell_{L}=0$ beam during nonlinear propagation from a beam waist $z=0$ to $z=3 z_{R}$ for FSL beams with input powers of $5 \mathrm{~mW}$ (purple), $7.5 \mathrm{~mW}$ (blue), $10.0 \mathrm{~mW}$ (green), $15.0 \mathrm{~mW}$ (orange) and $50.0 \mathrm{~mW}$ (red).

As before, the size of the FSL beam can also be used to control the Rayleigh range. In Fig. 8 we plot the polarization rotation during nonlinear propagation from a beam waist $z=0$ to $z=3 z_{R}$ for FSL beams with waists of $100 \mu \mathrm{m}$ and $\Delta\left|\ell_{R, L}\right|=1,2$ (dark blue, cyan) using 12 with $P=7.44 \mathrm{~mW}$ and $n_{2}=8 \times 10^{-6} \mathrm{~W} \mathrm{~cm}^{-2}$ s.t. $\mu=257.5$ and $\sigma=19.8$. Equivalent results for beams with waists of $200 \mu \mathrm{m}$ (green, magenta) and with rescaled experimental parameters $P=29.8 \mathrm{~mW}$ and $n_{2}=2 \times 10^{-} 6 \mathrm{~W} \mathrm{~cm}^{-2}$ s.t. $\sigma$ and $\mu$ are unchanged. We consider two superpositions of FSL beams $\left(\ell_{R}=1, \ell_{L}=0\right.$ and $\left.\ell_{R}=2, \ell_{L}=1\right)$ and show that the results for the two different beam waists match in both cases if we plot rotation as versus number of Rayleigh lengths propagation (Fig. 8(left)). If we polt the same results but as a function of distance ((Fig. 8 (left))) we can see that by doubling the beam waist, and hence increasing $z_{R}$ by a factor 4 , the rotation of the larger beam is correspondingly decreased by a factor $1 / 4$.
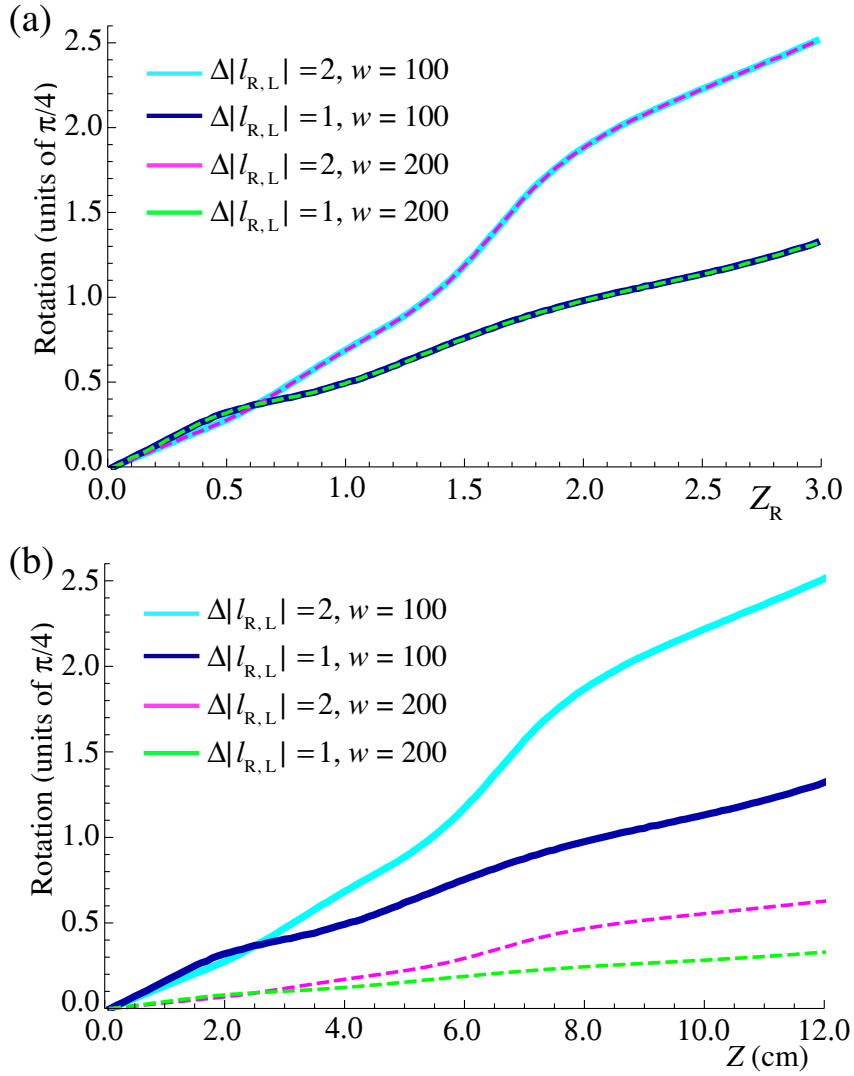

FIG. 8: Polarization rotation during nonlinear propagation for FSL beams with $\Delta\left|\ell_{R, L}\right|=1,2$ and waists of $100 \mu \mathrm{m}$ (dark blue, cyan) and $200 \mu \mathrm{m}$ (green, magenta). In both cases $\mu=257.5$ and $\sigma=19.8$. (a) rotation versus number of Rayleigh lengths, (b) rotation versus distance $(\mathrm{cm})$.

Biased Vector Beams $-\gamma \neq \pi / 4$

While many applications of cylindrical vector beams use spatially-varying linear polarization (radial or azimuthal), beams with azimuthally-varying elliptical polarization allow the space-variant spin in the beam to be transferred to the atomic medium, thus offering a unique way to manipulate atoms spatially [12]. Such elliptically polarized FSL beams can be produced by changing the relative amplitudes of the two eigenmodes of a cylindrical vector beam (i.e. by changing the value of $\gamma$ in Eq. 2). As we have already mentioned, equal-amplitude $\mathrm{CV}$ beams do not experience any polarization rotation during propagation - linearly or nonlinearly as both components have the same magnitude of OAM, and hence experience the same Gouy phase and the same spatial intensity distribution. However, when elliptically polarized, or biased, $\mathrm{CV}$ beams are propagated in the nonlinear medium we find that the polarization distributions do rotate and that the amount and direction of the rotation is dependent on the ellipticity of the polarization. This is clearly shown in Fig. 9 where $\left|\ell_{R}\right|=\left|\ell_{L}\right|=1$ and $\gamma$ in Eq. 2 has been chosen s.t. $\left|E_{R}\right|:\left|E_{L}\right|$ is $4: 1$ (red), $2: 1$ (blue), $1: 1$ (green), $1: 2$ (cyan), 
$1: 4$ (magenta). Note that as the bias is increased the beams

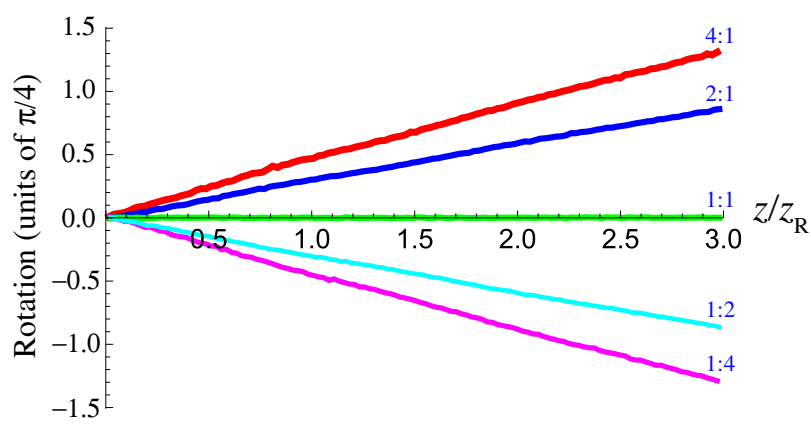

FIG. 9: Nonlinear polarization rotation for propagation of a cylindrical vector beam with $\left|\ell_{R, L}\right|=1$ over $3 z_{R}$. The parameter $\gamma$ in 2 is chosen s.t. $\left|E_{R}\right|:\left|E_{L}\right|$ is $4: 1$ (red), $2: 1$ (blue), $1: 1$ (green), $1: 2$ (cyan), $1: 4$ (magenta).

become more "scalar" as the contribution of the second beam becomes negligible, and the polarization becomes circular. In this case we recover the behaviour demonstrated in [14] where the scalar beam is shown to fragment more quickly than the corresponding vector beam. As Fig. 10 shows, the amount of rotation saturates as the bias increases. The rotation may also be increased by using higher-order $\mathrm{CV}$ beams, but as these diffract more for the same experimental parameters, a higher nonlinearity is required in order to keep the beams confined.

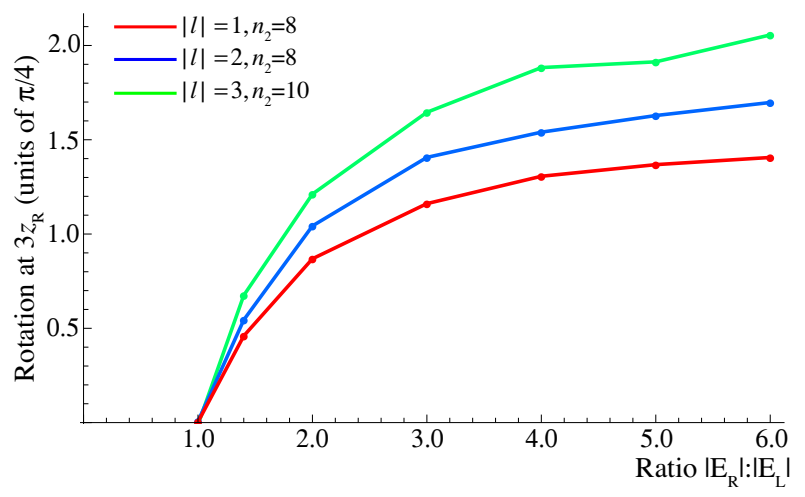

FIG. 10: Nonlinear polarization rotation at $3 z_{R}$ as a function of bias for biased CV beams with $\left|\ell_{R, L}\right|=1,2,3$ (red, blue, green).

One effect of this rotation is the conversion of radial (or azimuthal) elliptical polarization to azimuthal (or radial) with minimal changes to the spatial profile of the beam, as shown in Fig. 11. By choosing the bias and the propagation distance for a given beam waist we can control the polarization of the beam with no polarization optics.

\section{CONCLUSION}

Knowing how the spatial polarization distribution of a beam is affected by propagation is of importance in many applica-

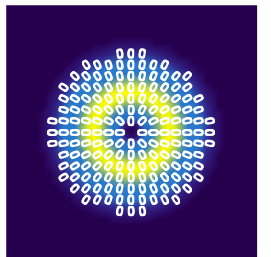

$z=0$

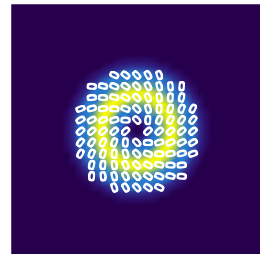

$z=1.3 z_{\mathrm{R}}$

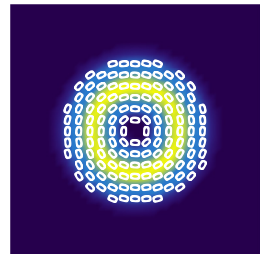

$z=2.5 z_{\mathrm{R}}$
FIG. 11: Radially elliptically polarized vector beam with $\left|\ell_{R, L}\right|=1$ and $\left|E_{R}\right|:\left|E_{L}\right|=4: 1$. Left to right: At a beam waist $z=0$, after nonlinear propagation over $z=1.3 Z_{R}, z=2.5 Z_{R}$. There is little change to the spatial profile of the beam during propagation, but the polarization changes from radial through spiral to azimuthal.

tions that depend on the state of polarization. We have analytically calculated the polarization rotation of fully-structured light (FSL) beams during linear propagation and shown that the observed rotation is due entirely to the difference in Gouy phase between the two eigenmodes comprising the FSL beams. This allows the exact polarization state at a particular propagation distance to be controlled simply by choosing the eigenmodes of the FSL beam and the beam size. Moreover, we have shown that polarization rotation is also affected by propagation through a self-focussing (Kerr) nonlinear medium and that this can be controlled by changing the eigenmodes of the superposition, and physical parameters such as the beam size, the amount of Kerr nonlinearity and the input power. In addition to the ability to control both the intensity and polarization of FSL beams may provide a useful method for applications in micromachining and microscopy [4, 34]. Finally, we have shown that by biasing cylindrical vector $(\mathrm{CV})$ beams to have elliptical polarization, we can vary the polarization state from radial through spiral to azimuthal using nonlinear propagation.

\section{ACKNOWLEDGEMENTS}

We would like to acknowledge support from the EPSRC DTA grant EP/M506643/1 and from The Carnegie Trust for the Universities of Scotland. AMY would like to thank The Leverhulme Trust for the award of a Leverhulme Trust Research Project Grant to further support this research. 
* Electronic address: alison.yao@ strath.ac.uk

[1] Q. Zhan, Adv. Opt. Photonics 1, 1-57 (2009).

[2] A. M. Beckley, T. G. Brown, and M. A. Alonso, Opt. Express 18, 10777 (2010).

[3] E. J. Galvez, S. Khadka, W. H. Schubert, and S. Nomoto, App. Opt. 51, 2925 (2012).

[4] A. V. Nesterov and V. G. Niziev, J. Phys. D: Appl. Phys. 33, 1817 (2000).

[5] K. I. Willig, S. O. Rizzoli, V. Westphal, R. Jahn and S. W. Hell, Nature 440, 935 (2006).

[6] P. Török and P. R. T. Munro, Opt. Express 12, 3605 (2004).

[7] K. S. Youngworth and T. G. Brown, Opt. Express 7, 77 (2000).

[8] S. N. Khonina and I. Golub, J. Opt. Soc. Am. A. 29, 2242 (2012).

[9] L. Novotny, M. R. Beversluis, K. S. Youngworth, and T. G. Brown, Phys. Rev. Lett. 86, 5251 (2001).

[10] A. LaPorta and M. D. Wang, Phys. Rev. Lett. 92, 190801 (2004).

[11] B. Sick , B. Hecht and L. Novotny, Phys. Rev. Lett. 85, 4482 (2000).

[12] F. K. Fatemi, Opt. Express 19, 25143 (2011).

[13] N. Bozinovic, Y. Yue, Y. Ren, M. Tur, P. Kristensen, H. Huang, A.E. Willner and S. Ramachandran, Science, 340, 1545, (2013).

[14] F. Bouchard, H. Larocque, A. M. Yao, C. Travis, I. De Leon, A. Rubano, E. Karimi, G.-L. Oppo, and R. W. Boyd, Phys. Rev. Lett. 117, 233903 (2016).

[15] C. Gabriel, A. Aiello, W. Zhong, T. G. Euser, N. Y. Joly, P. Banzer, M. Förtsch, D. Elser, U. L. Andersen, Ch. Marquardt, P. St.J. Russell, and G. Leuchs, Phys. Rev. Lett. 106, 060502 (2011).

[16] X.-F. Qian and J. H. Eberly, Opt. Lett. 36, 4110 (2011).

[17] S. Berg-Johansen, F. Töppel, B. Stiller, P. Banzer, M. Ornigotti,
E. Giacobino, G. Leuchs, A. Aiello and Ch. Marquardt, Optica 2, 864 (2015).

[18] R. Dorn, S. Quabis, and G. Leuchs, Phys. Rev. Lett. 91, 233901 (2003).

[19] P. Serafim, P. Sprangle and B. Hafizi, IEEE Trans. Plasma Sci. 28, 1155 (2000).

[20] R. Y. Chiao, E. Garmire and C. H. Townes, Phys. Rev. Lett. 13, 479 (1964)

[21] W. J. Firth and D. V. Skryabin, Phys. Rev. Lett. 79, 2450 (1997).

[22] A. S. Desyatnikov and Y. S. Kivshar, Phys. Rev. Lett. 87, 033901 (2001).

[23] M. S. Bigelow, Q. Han. Park, and R. W. Boyd, Phys. Rev. E 66, 046631 (2002).

[24] M. S. Bigelow, P. Zerom, and R. W. Boyd, Phys. Rev. Lett. 92, 083902 (2004).

[25] A. S. Desyatnikov, D. Mihalache, D. Mazilu, B. A. Malomed, and F. Lederer, Phys. Lett. A 364, 231 (2007).

[26] S. M. Barnett and R. Zambrini in Quantum Imaging, K. I. Kolobov, ed., (Springer, Singapore, 2007), pg. 284.

[27] R. W. Boyd, J. Opt. Soc. Am. 70, 877 (1980).

[28] D. N. Pattanayak and G. P. Agrawal, Phys. Rev. A 221159 (1980).

[29] A. V. Volyar, V. G. Shvedov, and T. A. Fadeeva, Opt. Spectrosc. 90, 93 (2001).

[30] M. R. Dennis, K. O'Holleran and M. J. Padgett, Progress in Optics 53, 293 (2009).

[31] B. E. A Saleh and M. C. Teich, "Fundamentals of Photonics", Wiley (2007).

[32] G. Cincotti, A. Ciattoni and C. Sapia, Opt. Commun. 220, 33 (2003).

[33] A. Ciattoni, B. Crosignani, P. Di Porto, and A. Yariv, Phys. Rev. Lett, 94, 073902 (2005)

[34] K. J. Mitchell, S. Turtaev, M. J. Padgett, T. Čižmàr, and D. B. Phillips, Opt. Express 24, 29269 (2016). 